\title{
GaN quantum dot based quantum information/computation processing
}

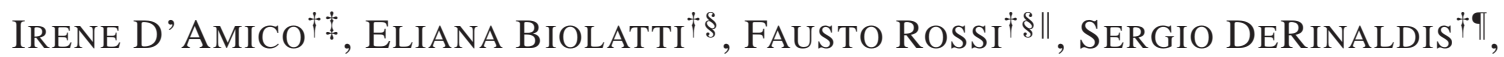

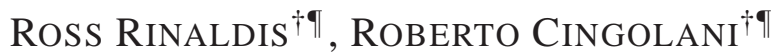 \\ ${ }^{\dagger}$ Istituto Nazionale per la Fisica della Materia (INFM) \\ ${ }^{\ddagger}$ Institute for Scientific Interchange (ISI), Villa Gualino, Viale Settimio Severo 65, 1-10133 Torino, Italy \\ ${ }^{\S}$ Dipartimento di Fisica, Politecnico di Torino, Corso Duca degli Abruzzi 24, 1-10129 Torino, Italy \\ II National Nanotechnology Laboratory (NNL), Università di Lecce, via per Arnesano, Lecce, Italy
}

(Received 11 March 2002)

\begin{abstract}
In this paper we propose to use GaN quantum dots (QDs) as building blocks for solid state quantum computing devices. The existence of a strong built-in electric field induced by the spontaneous polarization and by the piezoelectricity is exploited to entangle few-exciton states in coupled QDs without the use of external fields. The analysis of the electro-optical response of the coupled GaN QDs is based on a realistic-i.e. fully tri-dimensionaldescription of Coulomb-correlated few-electron states, obtained via a direct-diagonalization approach. The combined effect of the built-in electric field and ultrafast sequences of multicolor laser pulses in the few-carrier regime is investigated. We show how the built-in field induces intrinsic dipole-dipole coupling and thus allows the implementation of quantum information processing. As an example we will implement basic quantum information gates and we will demonstrate that our implementation scheme is compatible with a subpicosecond operation timescale, i.e. with timescales much lower than the decoherence time of the system.
\end{abstract}

(c) 2002 Elsevier Science Ltd. All rights reserved.

Key words: quantum dot, quantum computation.

\section{Introduction}

In recent years large interest has been focused by the scientific community on quasi-zero-dimensional (0D) systems, called semiconductor quantum dots (QDs) [1]. Compared to systems of higher dimensionality-like quantum wells and wires-they have a discrete, i.e. atomic-like, energy spectrum and, more importantly, they exhibit genuine few-carrier effects. This implies a radical change in the theoretical schemes [2] as well as in the experimental techniques [3] used to study such quasi 0D nanostructures, often referred to as semiconductor macroatoms. Apart from their relevance in terms of basic physics, these novel semiconductor nanostructures have attracted general attention because of their technological applications; these range from laser emitters [4] to charge-storage devices [5], from fluorescent biological markers [6] to quantum information processing devices [7]. In QDs, the flexibility typical of semiconductors in controlling carrier densities

"E-mail: frossi@athena.polito.it 
has been brought to its extreme: it is possible to electrically inject single electrons [8] or to photo-generate in a QD a single Coulomb-correlated electron-hole pair, i.e. a single exciton [9, 10]. It is even possible to detect the single-exciton decaying energy emission $[9,10]$. The quantized, atomic-like, energy structure of QDs allows for a rich optical spectrum and for a weak interaction of the QD system with environmental degrees of freedom (like phonons, plasmons, etc.), i.e for an almost decoherence-free quantum evolution of the carrier subsystem $[11,12]$. Moreover, their reduced spatial extension-up to few nanometers-leads to an increase of two-body interactions among carriers and to stronger Coulomb-correlation effects [2]. The latter may be used to design a variety of single-electron devices. In particular, they can be employed to design fully-optical quantum gates, as recently proposed in [12].

However, one of the major problems in such solid state quantum bits, is the need of an external electric field to implement the two-qubit gate. From the technological point of view this requires the presence of electrical contracts which limits the time response of the system and strongly complicate the physical interconnection of the device. The ideal scheme would thus be a QD system with built-in electric field. GaN-based QDs seem to match quite well these requirements [13], as they are known to exhibit strong built-in polarization and piezoelectric field (of the order of $\mathrm{MV} \mathrm{cm} \mathrm{cm}^{-1}$ ).

In this paper we will analyze in detail the effects of this internal field and, in particular, we will investigate in depth the electro-optical properties and the exciton-exciton dipole interaction in coupled GaN QDs, with the aim of implementing a scheme for quantum information/computation (QIC) processing based on these nanostructures.

Due to the need of low decoherence rates, the first proposals for experimental realizations of quantum information processing devices originated from specialities in atomic physics [14], in quantum optics [15], and in nuclear and electron magnetic-resonance spectroscopy [16]. However, a realistic quantum computing hardware would require a large number of qubits, and this can hardly be achieved by such systems. In contrast, in spite of the serious difficulties related to the relatively 'fast' decoherence times, a solid-state implementation of QIC seems to be the only way to benefit from the recent progress in ultrafast optoelectronics [17] as well as in meso/nanostructure fabrication and characterization [1]. As originally envisioned in [11], gating of charge excitations could be performed by coherent-carrier-control, i.e. by exploiting present ultrafast laser technology [17], which allows one to generate and manipulate electron-hole quantum states on a subpicosecond timescale: [18]. In this respect, decoherence times on nano/microsecond scales can be regarded as 'long' ones. Based on this idea a few implementations have been recently put forward [19]. However, while in these proposals single-qubit operations are implemented by means of ultrafast optical spectroscopy, the control of two-qubit operations still involves the application of external fields and/or microcavity-mode couplings, whose switching times are much longer than decoherence times in semiconductor QDs. As already pointed out in [11], in order to take full advantage from modern ultrafast laser spectroscopy one should be able to design fully optical gating schemes able to perform single and two-qubit operations on a subpicosecond timescale.

As opposed to our previous proposal [12], in which GaAs-based QDs where proposed as building blocks of the qubit and a static external field was applied to the array of QDs to create dipole-dipole interactions, the advantage of GaN QDs is that dipoles are intrinsically induced by means of the built-in internal field [13]. We will show that the biexcitonic shift due to the dipole interaction is of the order of some meV allowing for subpicosecond quantum gate operations and we shall discuss the application of such field-induced few-exciton effects to design a semiconductor-based fully-optical quantum information processing strategy.

\section{Theoretical approach}

\subsection{Spontaneous polarization and piezoelectric field}

One of the most interesting features in GaN heterostructures, is the strong built-in electric field: it is induced both by the spontaneous polarization and by the piezo-electric field. Contrary to GaN quantum 
wells, where the major role is played by the spontaneous polarization charge accumulated at the GaN/AlGaN interfaces, while strain induced piezoelectric fields have a minor importance [20], in GaN QDs due to the non-central symmetry of the wurzite structure, piezoelectric effects become relevant. Moreover, since the dimensions of GaN and AlN unit cell differ slightly from those of an ideal hexagonal crystal, a spontaneous electrostatic polarization is present as well [13]. As shown in [13], the strain-induced piezoelectric potential and the spontaneous polarization contribution are of similar magnitude and of the same sign, both directed in the growth direction. The magnitude of the intrinsic electric field along the growth direction is almost the same inside and outside the dot, but it is opposite in sign.

One of the advantages of having a strong intrinsic electric field, instead of an external one as in [12], is that carriers remain trapped into the dot. In the external field case in fact, when a strong field is applied, carriers may escape from the dot, due to the final height of the lateral confinement.

The internal field in GaN QDs can be tailored using the size of the dot: the total electric field inside the dot is [20]:

$$
F_{d}=\frac{L_{b}\left(P_{\mathrm{tot}}^{b}-P_{\mathrm{tot}}^{d}\right)}{\varepsilon_{0}\left(L_{d} \epsilon_{b}+L_{b} \epsilon_{d}\right)}
$$

where $\epsilon_{b(d)}, P_{\text {tot }}^{b(d)}$ and $L_{b(d)}$ are respectively, the relative dielectric constant, the total polarization and the width of the barrier (QD). The field in the barrier $F_{b}$ is obtained by interchanging the indices $b$ and $d$. Equation (1) is derived for an alternating sequence of quantum wells and barriers, but we can consider it as a good approximation for our case, since we consider an alternating sequence of QDs and barriers in the growth $(z)$ direction. This approximation provides fields in very good agreement with the experimental findings of [21]. This approximation neglects the in-plane shape of the QD, but we can consider the lateral shape of the dot as to be simply responsible for the strong confinement of the electron and hole singleparticle wavefunctions, which present an in-plane spherical symmetry (see [13]). The effect of the lateral dot shape can then be properly taken into account by modelling the in-plane confining potential as an effective parabolic potential, since this preserves the symmetry of the wavefunctions. The polarization is the sum of the piezoelectric charge $P_{\text {piezo }}^{b(d)}$ induced by the lattice mismatch $(m s)$ and by the thermal strain $(t s)\left(P_{\text {piezo }}^{b(d)}=\right.$ $\left.P_{m s}^{b(d)}+P_{t s}^{b(d)}\right)$ and the spontaneous polarizability $P_{s p}^{b(d)}$ of the GaN/AIN interface: $P_{\text {tot }}^{b(d)}=P_{\text {piezo }}^{b(d)}+P_{s p}^{b(d)}$, where the subscript $b(d)$ indicates the barrier (QD).

The presence of the internal electric field induces dipoles in each QD when excitons are present.

This, in turn, will generate an inter-dot dipole-dipole coupling. For the case of two staked QDs $a$ and $b$ along the growth (z) direction (see Fig. 1) for which $\vec{p}_{i} \| \hat{z}, \vec{p}_{i}$, the dipole moment of the $i$-dot, we get:

$$
\Delta E=-2 \frac{p_{a} p_{b}}{Z^{3}},
$$

where $Z$ is the distance between the two dipoles.

\subsection{Few-particle system}

The physical system under investigation is composed by electron-hole pairs confined in an array of GaN QDs. Its total Hamiltonian can be regarded as the sum of two term,

$$
H=H^{\circ}+H^{\prime}=H^{\circ}+H_{c l}+H_{\mathrm{env}},
$$

where the term $H^{\circ}$ describes the correlated electron-hole subsystem, i.e. free carriers plus confinement potential plus carrier-carrier Coulomb interaction, and the term $H^{\prime}$ describes the interaction of the carrier subsystem with coherent-light sources $\left(H_{c l}\right)$ and environmental degrees of freedom $\left(H_{\mathrm{env}}\right)$, i.e. carrier-light plus carrier-phonon interactions. Let us focus on the first term and consider the gas of carriers, electrons $(e)$ and holes $(h)$ confined within the quasi-0D semiconductor struction. The QD is depicted as a parabolic in-plane potential plus a square-well like potential in the $z$ direction. As discussed in the previous section, the 


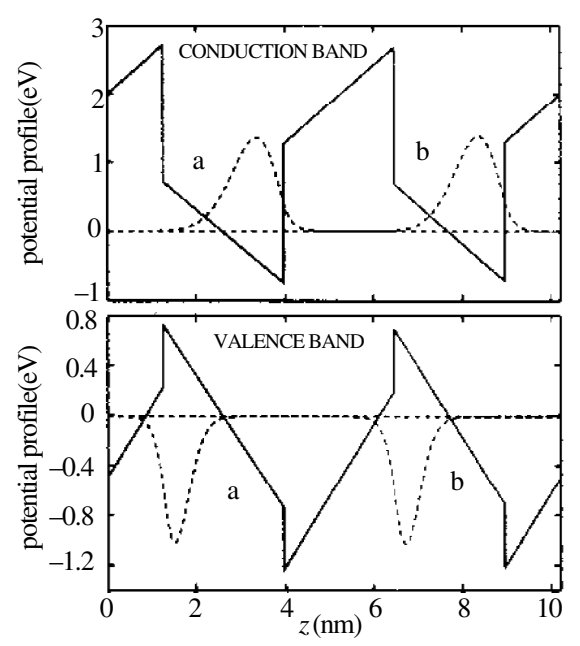

Fig. 1. Effects of the intrinsic electric field in a GaN-based QD structure; schematic representation of the square-well potential profile for electrons and holes along the growth direction modified by the internal field (solid lines) and single-particle spatial charge distributions for electrons (dashed curve, upper panel) and holes (dashed curve, lower panel).

effective in-plane confining potential takes into account the in-plane component of the built-in electric field, while the depth of the square-well is dictated by the conduction/valence band discontinuities. Our analysis is based on a realistic, fully three-dimensional description of the multi-QD structure. We get the many-body states describing a fixed number of electrons and holes in the structure by means of an exact diagonalization of the Hamiltonian, where we shall describe the bulk band structure in terms of the usual effective-mass approximation [22]. We shall work within the 'envelope-function' picture [23]. Concerning the carrier-carrier interaction terms, only processes conserving the total number of carriers are considered, thus Auger recombination and impact ionization are neglected. We can therefore look for many-body states $|\Psi\rangle$ corresponding to a given number of electrons $\left(N_{e}\right)$ and holes $\left(N_{h}\right)$. In particular, we shall consider the case of intrinsic semiconductor materials [22], for which $N_{e}=N_{h}$. For any given number of electron-hole pairs $N$, a direct diagonalization of $H_{\mathrm{o}}$ will provide the many-body states $\left|\lambda_{N}\right\rangle$ of the interacting electron-hole system; they, in turn, allow one to evaluate many-exciton optical spectra, i.e. the absorption probability corresponding to the generic $N \rightarrow N^{\prime}$ transition. Finally, the interaction of the carrier system with environmental degrees of freedom, like phonon and plasmon modes of the host material, is responsible for decoherence processes and it will be accounted for within a density-matrix formalism.

\section{Coupled QD structure}

The above theoretical scheme has been applied to realistic GaN QDs, stacked along the growth axis. The general scheme of our two-qubit quantum gate, consists of two stacked dots $a$ and $b$ of slightly different size. Two dot systems grown epitaxially can be tailored in such a way that the seeding dot is smaller than the top-most dot, due to the overlap of the strain fields, or the two dots have identical base diameter but slightly different height, thanks to the control in the deposition rate and III-V ratio. In both cases the potential of the two dots is engineered to control the splitting of the respective ground levels, thus allowing selective optical excitation of one of the two dots by ultrafast laser pulses.

For the sake of clarity, we discuss the situation of two dots with identical bases, stacked along the growth axis, and having heights of 2.5 and $2.7 \mathrm{~nm}$, respectively. Analogous results would be obtained by two dots of same height and different base-radius. The resulting difference in the potential well width $w_{d}$ of each QD allows to slightly shift the excitonic transitions $\left(\delta w_{d} \simeq 0.2 \mathrm{~nm}\right)$. The barrier width $w_{b}$ is such to prevent 
single particle tunneling but to allow at the same time significant dipole-dipole Coulomb coupling. We evaluated the internal electric field according to eqn (1), where $P_{\mathrm{ms}}^{b(d)}=-2\left(\frac{e_{33} C_{13}}{C_{33}-e_{31}}\right) \sigma_{\|}^{b(d)}$ (all the material parameters can be found in [20], except for the lattice in-plane mismatch $\sigma_{\|}^{b}$ that is here $2.48 \%$ ), $P_{t s}^{d}=-3.2$. $10^{-4}, P_{t s}^{b}=0 \mathrm{C} \cdot \mathrm{m}^{-2}, P_{s p}^{b}=-0.081 \mathrm{C} \cdot \mathrm{m}^{-2}$ and finally $P_{s p}^{d}=-0.029 \mathrm{C} \cdot \mathrm{m}^{-2}$. The values used for $\sigma_{\|}^{b}$ and $P_{s p}^{b}$ take into account the increased percentage of $\mathrm{Al}(x=1)$ in the barrier in respect to [20] $(x=0.15)$.

Given these parameters, we have calculated, according eqn (1) the electric field for a $2.7 \mathrm{~nm}$ high dot and a $2.5 \mathrm{~nm}$ wide barrier obtaining $F_{d}=5.8 \mathrm{MV} \mathrm{cm}^{-1}$ for the barrier and $F_{d}=5.4 \mathrm{MV} \mathrm{cm}^{-1}$ in the dot. Starting from these results, and in order to take into account the slightly different size of the two coupled QDs, we used in our calculations the fields $F_{d}=5.6$ and $5.4 \mathrm{MV} \mathrm{cm}^{-1}$ for the two dots, and $F_{b}=5.7 \mathrm{MV} \mathrm{cm}{ }^{-1}$ for the barrier: these values allow for a potential profile that is periodic over the two-dot cell. The electric field strongly modifies the conduction-band and valence-band edges along the growth directions (see Fig. 1): this causes the separation of the carrier states and the creation of intrinsic dipoles as it can be seen in Fig. 1.

The electric field shifts in opposite directions the electron and the hole charges: electrons are forced to move towards the top of the dot and holes are driven towards its bottom. So, if we consider the two dots $a$ and $b$, and excite one exciton in each of them, we obtain two aligned dipoles which are both pointing in the same direction. As pointed out before, these results in the negative dipole-dipole coupling term eqn (2). The energy shift $\Delta E$ is opposite and its absolute value is doubled with respect to the configuration of [12], in which the two dipoles are also parallel but laying side by side.

We will now discuss the optical response of the semiconductor macromolecule $(a+b)$. The excitonic optical spectrum $(0 \rightarrow 1$ transition) in the presence of the intrinsic electric field is calculated by means of Fermi-Golden's rule and it is shown in Fig. 2 (solid curve). The two lowest optical transitions correspond to the formation of direct ground-state excitons in dot $a$ and $b$, respectively. Due to the strong built-in electric field in such heterostructures, the excitonic transitions energies are red-shifted and, as it can be seen in Fig. 2, the interband emission is fractions of ev below the GaN bulk bandgap energy. It is worth noticing that the difference in the ground state excitonic transition energies in each dot is one order of magnitude bigger than the corresponding energy difference in [12], and this is due to the presence of the strong intrinsic electric field in the growth direction. The two ground-state transitions have different amplitude, due to the non-symmetric structure we are considering. Since the two dots have different heights, the internal electric field which is experienced by the carriers in each dot is not the same. As a result the oscillator strength, i.e. the overlap of electron and hole single-particle wavefunctions, changes according to the specific dot. The peaks higher in energy correspond to optical transitions involving excited states of the in-plane parabolic potential in each QD. Moreover, since the electric field is in the growth direction, the in plane transition selection rules are conserved, in contrast to the scheme discussed in [12] where the in-plane static field was breaking the symmetry. As a consequence, in the case we are considering, only transitions conserving the single-particle momentum are allowed, so that the resulting absorption spectrum is simpler, with peaks that are in general better isolated and defined. This characteristic can become important when considering an energy-selective scheme to address the single transition, since it allows for shorter pulses, i.e. for a broader dispersion in energy of the pulses themselves. Due to the strong in-plane carrier confinement, the low-energy excitonic states closely resemble the corresponding single-particle ones (see the dashed curves in Fig. 2). As we can see, apart from a small rigid shift, corresponding to the exciton binding energy, the relative position of the lowest transitions is the same.

Let us now discuss the biexcitonic spectrum (solid curve in Fig. 3); it describes the generation of a second electron-hole pair in the presence of a previously created exciton ( $1 \rightarrow 2$ optical transitions). The crucial feature in Fig. 3 is the magnitude of the 'biexcitonic shift' [1], i.e. the energy difference between the excitonic and the biexcitonic transition (see solid and dashed curves). For the QD structure under investigation we get an energy splitting of $4.4 \mathrm{meV}$, which is comparable to the one in [12] and can be resolved by sub-picosecond energy pulses. 


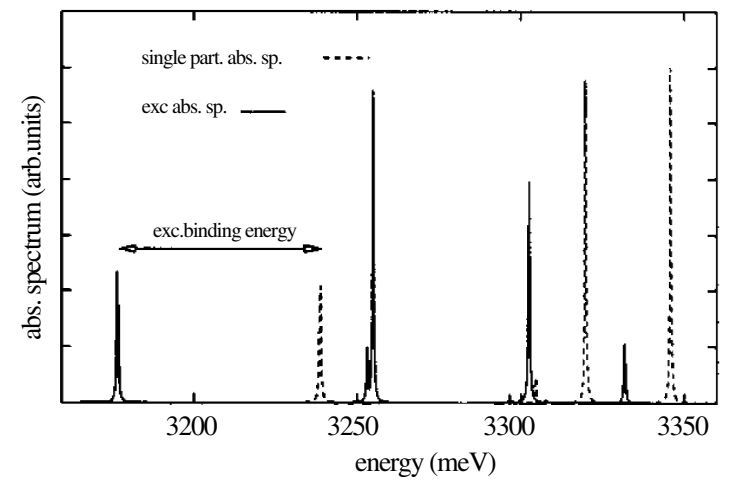

Fig. 2. Optical response of the GaN-based QD macromolecule of Fig. 1: excitonic (solid curve) absorption and single-particle spectrum (dashed curve).

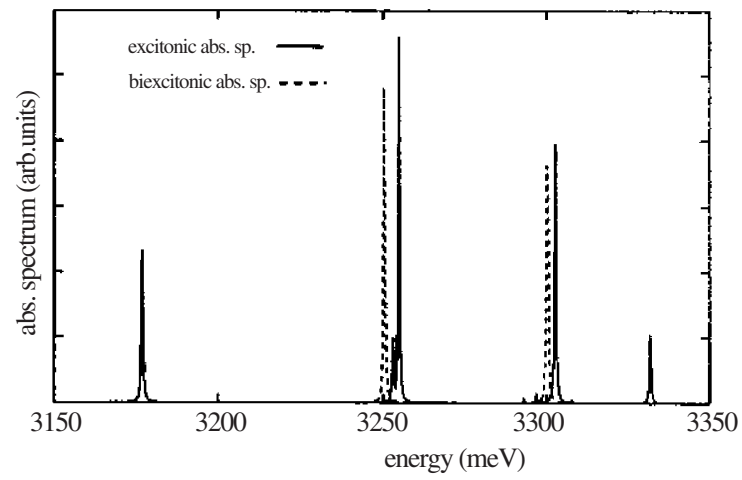

Fig. 3. Optical response of the GaN-based QD macromolecule of Fig. 1. Excitonic (solid curve) and biexcitonic absorption (dashed curve). The latter has been computed assuming as initial state an exciton in dot $a$ and the laser pulses are supposed to have the same circular polarization.

\section{Single and two qubit gates}

The core of our QIC proposal closely parallel the one presented in [12], that we shall now briefly review. The central idea is to exploit the biexcitonic shift to design conditional operations. We denote with $\hat{n}_{l}$ the excitonic occupation number operators, where $l$ indicates the generic QD in our array. The two states with eigenvalues $n_{l}=0$ and $n_{l}=1$ correspond, respectively, to the absence (no conduction-band electrons) and to the presence of a ground-state exciton (a Coulomb-correlated electron-hole pair) in dot $l$ : this will be our single-qubit basis: $|0\rangle_{l}$ and $|1\rangle_{l}$. The whole computational state-space is then spanned by the basis $|n\rangle=\otimes_{l}\left|n_{l}\right\rangle,\left(n_{l}=0,1\right)$.

The full many-body Hamiltonian $H_{\circ}$ in (3) restricted to the aforementioned computational space reduces to

$$
\tilde{H}_{\mathrm{o}}=\sum_{l} E_{l} \hat{n}_{l}+\frac{1}{2} \sum_{l l^{\prime}} \Delta E_{l l^{\prime}} \hat{n}_{l} \hat{n}_{l^{\prime}}
$$

where, $E_{l}$ denotes the energy of the ground-state exciton in dot $l$ while $\Delta E_{l l^{\prime}}$ is the biexcitonic shift due to the Coulomb interaction between dots $l$ and $l^{\prime}$, previously introduced (see Fig. 3). According to (4), the single-exciton energy $E_{l}$ is renormalized by the biexcitonic shift $\Delta E_{l l^{\prime}}$, induced by the presence of a second exciton in dot $l^{\prime}\left(\tilde{E}_{l}=E_{l}+\sum_{l^{\prime} \neq l} \Delta E_{l l^{\prime}} n_{l^{\prime}}\right)$. In order to better illustrate this idea, let us focus again on the two-QD structure, i.e. two-qubit system, and fix our attention on one of the two dots, say dot $b$. The 


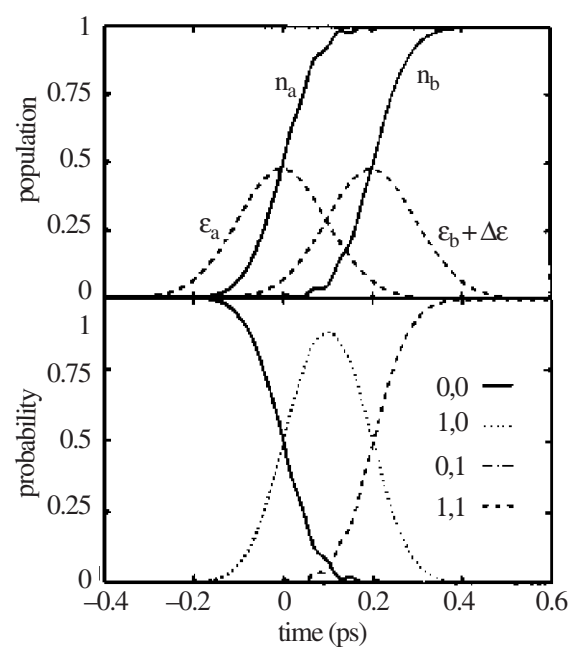

Fig. 4. Time-dependent simulation of a two-qubit operation realizing the first prescription $(|1,0\rangle \rightarrow|1,1\rangle)$ of a CNOT logic quantum gate in the coupled QD structure $a+b$ of Fig. 1 (see text). Exciton populations $n_{a}$ and $n_{b}$ (upper panel) and diagonal density-matrix elements (lower panel) as a function of time. The laser-pulse sequence is also sketched (dashed curve in the upper panel). For completeness the displayed simulation starts from the initial state $|1,0\rangle$.

effective energy gap between $|0\rangle_{b}$ and $|1\rangle_{b}$ depends now on the occupation of dot $a$. Exploiting this fact, we can design properly tailored laser-pulse sequences to implement controlled-not logic gates among the two QDs as well as single-qubit rotations. Indeed, by sending an ultrafast laser $\pi$-pulse with central energy $\hbar \omega_{b}\left[n_{a}\right]=E_{b}+\Delta E_{b a} n_{a}$, the transition $\left|n_{b}\right\rangle_{b} \rightarrow\left|1-n_{b}\right\rangle_{b}$ ( $\pi$-rotation) of the target qubit (dot $\left.b\right)$ is obtained if and only if the control qubit $(\operatorname{dot} a)$ is in the state $|n\rangle_{a}$.

Moreover, by denoting with $U_{b}^{n_{a}}$ the generic unitary transformation induced by the laser $\pi$-pulse of central frequency $\omega_{b}\left[n_{a}\right]$, it is easy to check that the two-color pulse sequence $U_{b}^{0} U_{b}^{1}$ achieves the unconditional $\pi$-rotation of qubit $b$.

We will now present the results of a few simulated experiments of basic quantum information processing, which demonstrate the viability of our proposal. Our time-dependent simulations are based on the realistic state-of-the-art QD structure of Fig. 1 for which $E_{a}=3.177 \mathrm{eV}, E_{b}=3.255 \mathrm{eV}, \Delta E=-4.4 \mathrm{meV}$. The simulations are based on a numerical solution of the Liouville-von Neumann equation describing the exact quantum-mechanical evolution of the many-exciton system (4) within our computational subspace in the presence of environment-induced decoherence processes [24]. Figure 4 shows a simulated sequence of twoqubit operations driven by two-color laser-pulses: this is the quantum conditional NOT gate: the state of the second QD is changed if and only if the first dot is in the state $|1\rangle$. We want to stress that the operation is performed on a sub-picosecond timescale, in particular the difference in time between the two $\pi$-pulses is only of 0.2 ps. Figure 5 shows instead the generation of an entangled state. Initially the system is in the state $|0,0\rangle \equiv|0\rangle_{a} \otimes|0\rangle$. The first laser pulse (at $t=0 \mathrm{ps}$ ) is tailored in such a way to induce a $\pi / 2$ rotation of the qubit $a:|0,0\rangle \rightarrow(|0,0\rangle+|1,0\rangle) / \sqrt{2}$. At time $t=0.4$ ps a second pulse induces a conditional $\pi$-rotation of the qubit $b:|0,0\rangle+|1,0\rangle \rightarrow|0,0\rangle+|1,1\rangle$. State entanglement plays a central role in any quantum information processing. The scenario described so far is confirmed by the time evolution of the exciton occupation numbers $n_{a}$ and $n_{b}$ reported in the upper panels of Fig. 4 (CNOT gate) and Fig. 5 (entanglement), as well as of the diagonal elements of the density matrix (in our four-dimensional computational basis, lower panel of the same figures). 


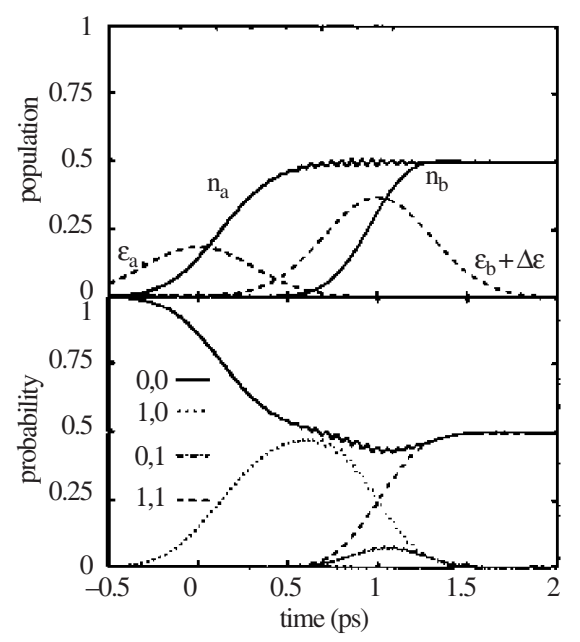

Fig. 5. Time-dependent simulation of a CNOT quantum gate transforming the factorized state $|0,0\rangle+|1,0\rangle$ into a maximally entangled state $|0,0\rangle+|1,1\rangle$ for the coupled QD structure $a+b$ in Fig. 1 (see text). Exciton populations $n_{a}$ and $n_{b}$ (upper panel) and diagonal density-matrix elements (lower panel) as a function of time. The laser-pulse sequence is also sketched (dashed curve in the upper panel). For completeness the displayed simulation starts from the initial state $|1,0\rangle$.

\section{Conclusions}

The experiment simulated above clearly shows that the energy scale of the biexcitonic splitting in our QD molecule (see Fig. 1) is compatible with the sub-picosecond operation timescale of Figs 4 and 5. It is worth summarizing the various advantages the intrinsic built-in electric field of nitride QDs carries. First of all, there is no need of applying external fields to the GaN dot array, and this allow an easier implementation of the quantum information proposal in respect to [12]. Moreover, the angular momentum in each allowed interband transition is conserved and the selection rules are now restored: this implies simpler spectra, and in turn makes the absorption spectra peaks easier to identify on the experimental side. Last but not least, due to the built-in electric field, the ground state excitonic transitions we are considering for quantum information processing are very well separated in energy, with an energy difference of about one order of magnitude with respect to [12].

An open question remains the technology and characterization of GaN QDs, but a lot of work is in progress both on the theoretical and on the experimental side: we hope that this purely technological limitation will be overcome in the near future.

In summary, we have proposed a scheme for implementing an all optical QIC process, which exploits the characteristic built-in electric field in GaN-based QDs. Our analysis has shown that energy-selected optical transitions in realistic state-of-the-art GaN QD structures are good candidates for quantum-information encoding and manipulation, and in particular the sub-picosecond timescale of ultrafast laser spectroscopy allows for a relatively large number of elementary operations within the exciton decoherence time. We additionally discussed the technological/experimental advantages of this scheme with respect to the one based on GaAs QDs.

\section{References}

[1] See, e.g., L. Jacak, P. Hawrylak, and A. Wojs, Quantum Dots (Springer, Berlin, 1998); D. Bimberg et al., Quantum Dot Heterostructures (Wiley, Chichester, 1998). 
[2] See, e.g., M. Rontani, F. Rossi, F. Manghi, and E. Molinari, Appl. Phys. Lett. 72, 957 (1998); Solid State Commun. 112, 151 (1999); U. Hohenester, F. Rossi, and E. Molinari, Solid State Commun. 111, 187 (1999).

[3] See, e.g., R. Rinaldi et al., Phys. Rev. B62, 1592 (2000).

[4] See, e.g., H. Saito et al., Appl. Phys. Lett 78, 267 (2001); O. Shchekin et al., Appl. Phys. Lett. 77, 466 (2000).

[5] See, e.g., J. J. Finley et al., Appl. Phys. Lett 73, 2618 (1998); G. Yusa and H. Sakaki, Appl. Phys. Lett 70, 345 (1994); T. Lundström et al., Science 286, 2312 (1999).

[6] See, e.g., M. Bruchez Jr et al., Science 281, 2013 (1998).

[7] See, e.g., D. Loss and D. P. DiVincenzo, Phys. Rev. A57, 120 (1998); M. Sherwin et al., Phys. Rev. Lett. A60, 3508 (1999); T. Tanamoto, Phys. Rev. A61, 22305 (2000).

[8] See, e.g., B. T. Miller et al., Phys. Rev. B56, 6764 (1997); S. Tarucha et al., Phys. Rev. Lett. 77, 3613 (1996); E. Deckel et al., Phys. Rev. Lett. 80, 4991 (1998); L. Landin et al., Science 280, 262 (1998); U. Banin et al., Nature 400, 542 (1999); F. Findeis et al., Solid State Commun. 114, 227 (2000).

[9] Bayer et al., Phys. Rev. B58, 4740 (1998); U. Banin et al., Nature 400, 542 (1999).

[10] P. Hawrylak et al., Phys. Rev. Lett. 85, 389 (2000); M. Bayer et al., Nature 405, 923 (2000).

[11] P. Zanardi and F. Rossi, Phys. Rev. Lett. 81, 4752 (1998).

[12] E. Biolatti, R. C. Iotti, P. Zanardi, and F. Rossi, Phys. Rev. Lett. 85, 5647 (2000).

[13] R. D. Andreev and E. O. O’Reilly, Phys. Rev. B62, 15851 (2000); F. Widmann et al., Phys. Rev. B58, R15989 (1998).

[14] J. I. Cirac and P. Zoller, Phys. Rev. Lett. 74, 4091 (1995); T. Pellizzari et al., Phys. Rev. Lett. 75, 3788 (1995); C. Monroe et al., Phys. Rev. Lett. 75, 4714 (1995); A. Sorensen and K. Molmer, Phys. Rev. Lett. 82, 1971 (1999).

[15] Q. A. Turchette et al., Phys. Rev. Lett. 75, 4710 (1995); A. Imamoglu et al., Phys. Rev. Lett. 83, 4204 (1999).

[16] N. Gershenfeld and I. Chuang, Science 275, 350 (1997).

[17] See, e.g., J. Shah, Ultrafast Spectroscopy of Semiconductors and Semiconductor Nanostructures (Springer, Berlin, 1996).

[18] N. H. Bonadeo et al., Science 282, 1473 (1998).

[19] M. Sherwin et al., Phys. Rev. A60, 3508 (1999); T. Tanamoto, Phys. Rev. A61, 22305 (2000).

[20] R. Cingolani et al., Phys. Rev. B61, 2711 (2000).

[21] F. Widmann et al., Phys. Rev. B58, 15989 (1998).

[22] See, e.g., P. Y. Yu and M. Cardona, Fundamentals of Semiconductors (Springer, Berlin, 1996).

[23] See, e.g., G. Bastard, Wave Mechanics of Semiconductor Heterostructures, Les Editions de Physique (Les Ulis, France, 1989).

[24] Decoherence processes are accounted for in our density-matrix formalism by means of a standard $T_{1} T_{2}$ $\operatorname{model}\left(T_{1}=1 \mathrm{~ns}, T_{2}=50 \mathrm{ps}[18]\right)$. 